\title{
El campo de los estudios urbanos y regionales desde el Sur: anotaciones acerca de los desafíos teóricos y las posibilidades de una reconstrucción teórico-metodológica crítica en la periferia del capitalismo
}

Carlos Brandáo. Universidad Federal de Río de Janeiro (UfrJ), Río de Janeiro, Brasil.

RESUMEN | Existe un amplio patrimonio intelectual acumulado que busca interpretar las configuraciones socioespaciales, basándose para ello en diversos paradigmas teóricos. No ignorando ese legado, este artículo propone un abordaje crítico y alternativo que procura interpelar ese importante conocimiento heredado, levantando algunas problematizaciones y formulando ciertas categorías de análisis puntuales. Tal planteamiento intenta contribuir en la aprehensión de las variadas dimensiones espaciales de los procesos de reproducción de la vida social, pensados histórica y concretamente. La propuesta se concentra en construir mediaciones teóricas e históricas más pertinentes a los estudios urbanos y regionales, estimulando un debate horizontal entre el pensamiento crítico histórico-estructural del Sur con las interpretaciones de la producción social del espacio del Norte. El artículo se concentra en presentar una reformulación con una perspectiva crítica y enunciada desde América Latina.

PALABRAS CLAVE | teoría del desarrollo regional, planificación territorial, transformaciones socioterritoriales.

ABSTRACT | There is a wide range and diverse accumulated intellectual heritage that seeks to decipher and interpret socio-spatial configurations. That rich scientific legacy has been guided and developed by various currents and theoretical paradigms. Not ignoring that legacy, this article proposes a critical and alternative approach that seeks to challenge that important inherited knowledge with some problematizations, formulating some categories of analysis. Such an approach, based on different analytical planes and levels of abstraction, tries to contribute to the apprehension of the various spatial dimensions of the processes of reproduction of social life. The proposal focuses on the challenges of the theoretical construction, and historical mediations pertinent to urban and regional studies, and which engage in a dialogue between the Latin American historical-structural thinking, and the social production of space of the interpretations of the North. It suggests a stimulating frame of critical debate, enunciated from the South.

KEYWORDS | regional development theory, territorial planning, socio-territorial transformations.

Recibido el 31 de marzo de 2020, aprobado el 19 de mayo de 2020.

E-mail: BrandaoUFRJ@gmail.com 


\section{Introducción}

Un sencillo homenaje a Carlos de Mattos, Alejandro Rofman y José Luis Coraggio.

En la muy particular coyuntura de profunda y multidimensional crisis (económica, política y sanitaria) en que estamos inmersos, las reflexiones sobre la organización espacial urbano-regionales son cruciales.

En el año 2020 se completó más de medio siglo de construcción de pensamiento crítico en el campo del conocimiento que busca aprehender la dinámica de los procesos sociales que producen las sociedades de manera disputada, relacional y conflictiva en el espacio.

Existe una serie de corrientes teóricas que estructuran diferentes preguntas por medio de distintas categorías y perspectivas de análisis. Esta producción científica transcurre entre permanentes disputas y contrapuntos. Esas contiendas -que no son triviales, dada la complejidad y la pluridimensionalidad de los fenómenos socialesexigen múltiples miradas, dado el objeto complejo que define la producción espacial a través de las relaciones sociales.

En este artículo planteamos que debemos partir del reconocimiento de un amplio y muy rico patrimonio intelectual acumulado por cerca de doscientos años de reflexiones. Esa herencia académica tiene su origen en las contribuciones más generales que están presentes en algunos de los clásicos del pensamiento social que tuvieron una mayor sensibilidad con la dimensión espacial de los procesos sociales, políticos y económicos.

Por otro lado, si tomamos el período que se inicia con las revueltas sociales de 1968 en Europa y en los Estados Unidos de América, como el momento de una reacción más organizada que enfrenta al pensamiento conservador aún vigente en el área (a partir de las obras seminales de Castells, 1972; Harvey, 1973; Lefebvre, 1968), completamos cincuenta años de una contundente elaboración teórica y política, la cual tiene por base un abordaje crítico de la realidad que busca construir una interpretación de la producción social del espacio. Sin embargo, al ser tal abordaje formulado básicamente en Europa y en los Estados Unidos, poco o nada de él se concentra en la situación específica de los espacios subdesarrollados y periféricos del sistema capitalista.

No obstante, por su lado, América Latina sí ha elaborado, de forma autónoma y original, por cerca de setenta años -desde finales de la década de 1940-, un patrimonio académico propio para pensar las especificidades del proceso de subdesarrollo en la condición periférica, ${ }^{1}$ investigando las heterogeneidades estructurales y los diferentes estilos de desarrollo presentes en el continente. En ese sentido, entre los aportes realizados por el pensamiento histórico-estructural latinoamericano

1 A lo largo de todo ese artículo, cada vez que utilizamos la expresión "países periféricos" para caracterizar la situación de América Latina, preferiríamos retomar una concepción más completa, una que definiese la naturaleza simultáneamente cuádrupla de esa situación, según la formulación más precisa de Florestan Fernandes (1972), que afirma que son en verdad "países periféricos, subdesarrollados, dependientes e imperializados". 
encontramos también un cierto diálogo más directo con la dimensión espacial, que precisa ser valorizado y ampliado.

Ahora bien, estos dos grandes campos del pensamiento no deben ser olvidados, necesitamos retomarlos y reactualizarlos. Debemos avanzar en el diálogo entre esas formulaciones situadas desde el Sur, y las concepciones de producción social del espacio elaboradas desde el Norte, buscando articular ambos campos y mirando, al mismo tiempo, las transformaciones geopolíticas, geoeconómicas y geoculturales al calor de la crisis del capitalismo del siglo XxI.

El legado de estos dos marcos intelectuales críticos nos enseña cómo el rescate de la problemática de la reproducción de las clases sociales, el rol del Estado y de los encuadramientos históricos se vuelven cruciales para el entendimiento de las dimensiones urbanas y regionales del proceso de desarrollo desigual capitalista. De igual manera, vale aclarar que no desconocemos los enormes desafíos que existen para recolocar en el centro del debate de las ciencias sociales y humanas la cuestión de las relaciones entre espacio, capital, trabajo, clases sociales y Estado.

Más allá de ese reposicionamiento necesario, es preciso construir robustas y más definidas mediaciones históricas y teóricas que interpelen simultáneamente las transformaciones del capitalismo contemporáneo, junto con las especificidades de sus espacios periféricos, a fin de pensar la producción social del espacio y su transformación.

Sería deseable en esa empresa procurar un cuadro de referencia lo suficientemente amplio como para permitir la formulación de preguntas pertinentes sobre estos procesos, abarcando desde los más generales hasta los más específicos, para así poder avanzar en investigaciones empíricas específicas en los espacios urbano-regionales.

Esta propuesta, de naturaleza ensayística y exploratoria, plantea el objetivo de ofrecer un panorama general de los desafíos teóricos y analíticos en el campo del planeamiento regional y urbano, apuntando, muy sucintamente, las posibilidades de una reconstrucción teórico-metodológica crítica. Esta invitación, además, debe ser tomada como una agenda amplia, que demanda incluir a las pesquisas en curso, para que pueda ser desarrollada y robustecida en los próximos años. En este sentido, se intenta contribuir en el planteamiento de un esquema analítico de orden general que pueda ser enriquecido poco a poco por posteriores estudios específicos, concretos y empíricos. Asimismo, realizamos un mapeo general de los autores más relevantes, aunque, de igual manera, los límites naturales de este artículo impiden profundizar en sus contribuciones específicas. Sin embargo, consideramos importante mencionarlos y puntuar su existencia, junto con demarcar las líneas más importantes, las trayectorias y las tendencias de los debates de nuestro campo de conocimiento.

El artículo está dividido en cuatro partes, además de la introducción. Primero, presentamos una muy sintética memoria genealógica de las principales formulaciones teóricas acerca de la organización del espacio. En seguida, discutimos sucintamente las cuatro corrientes teóricas que estudian el espacio. Presentamos en la tercera parte un esquema teórico, que se estructura en tres categorías de análisis, cada una de ellas con cuatro elementos constituyentes. Estas tres categorías de análisis son: i) las cuatro dimensiones espaciales de la división social del trabajo (interramificaciones, interregionalidades, interurbanidades, interacciones rivales interpersonales); ii) los 
cuatro poderes transformadores del espacio social (privado, público, fuerzas contrahegemónicas, micropoderes); y iii) los cuatro planos o niveles de análisis (escalas espaciales, niveles de gobierno, ámbitos y arenas de poder, prácticas espaciales de la vida cotidiana).

Finalmente, en el cuarto y último apartado, presentamos las conclusiones. Allí puntuamos algunos interrogantes y apuntamos una agenda para futuras investigaciones.

\section{Breve memoria genealógica de las formulaciones teóricas acerca de la organización del espacio}

Cualquier tentativa de rescatar del olvido y puntear las cuestiones clave en torno a las reflexiones del pensamiento social que hayan conseguido establecer relaciones entre la organización social y sus dimensiones espaciales, es muy arriesgada y ciertamente quien lo intente paga su precio. Sin embargo, son innúmeros los autores y las corrientes teóricas que en los últimos doscientos años buscaron investigar el papel del espacio en el contexto general de la estructura y de la dinámica social. Ese patrimonio intelectual acumulado por tan largo periodo no puede ser ignorado o despreciado cuando se busca pensar las cuestiones socioespaciales contemporáneas.

La preocupación por la dimensión espacial del proceso de desarrollo capitalista aparece ya en algunos de los clásicos del pensamiento social, entre los cuales destacan las obras de Willian Petty (1623-1687), Élienne Condillac (1714-1780), Adam Smith (1723-1790), Simonde de Sismondi (1773-1842), junto a muchos otros. Con todo, es el libro The Isolated State, de 1826, de Johann Von Thünen (1783-1850), el que se suele utilizar para señalar el inicio de este campo especializado del conocimiento.

En este trabajo, lamentablemente, no tenemos espacio para realizar una genealogía más profunda de ese rico y complejo marco de pensamiento. Sin embargo, disponemos de amplias y profundas sistematizaciones de esa vasta literatura, por ejemplo en Capel (1981), Castro et al. (1995), Furió (1996), Gottdiener (1997), Méndez (1997), Lencioni (1999), Moraes (2000), Riffo (2013), Paasi et al. (2018) y Haesbaert (2019).

Son muy variadas y amplias las contribuciones de los clásicos que formularon o institucionalizaron en las ciencias sociales el diálogo con las problemáticas espaciales. Para comenzar, empezamos por destacar, por un lado, los aportes de Paul Vidal de la Blache (1845-1918) y Friedrich Ratzel (1844-1904); y por el otro, los de la Escuela de Chicago. En este punto es necesario exponer que somos conscientes de que existe una gran controversia en torno a los planteamientos de tales pioneros. No obstante, asumimos esa responsabilidad y arriesgamos un intento de síntesis, tal vez excesivamente concisa, como la que aquí exponemos. Sin duda, claro está, quedarán muchas lagunas y deficiencias; de ahí que solamente se enfaticen algunos puntos que consideramos imprescindibles para el desarrollo de las argumentaciones más generales de este ensayo.

Los dos primeros, La Blache y Ratzel, plantearon cuestiones fundantes de la geografía humana, como las relaciones humano-naturaleza, y el rol de la política, 
del Estado, de la región y del territorio. Son autores que son fuente de grandes polémicas y diversas lecturas, algunas veces restrictivas y simplificadoras. Estos científicos inclusive quedaron rotulados respectivamente como padres de las escuelas "francesa" o "posibilista/voluntarista", y "alemana" o "determinista".

Paul Vidal de la Blache estructura la agenda de lo que se constituirá en las principales temáticas de las discusiones espaciales, tales como paisaje, región y población asentada. En la concepción de este autor, los seres humanos aparecen ocupando espacios específicos, actuando e interactuado con la naturaleza, movilizando técnicas y tomando decisiones, envueltos en particulares contextos históricos y geográficos y abiertos a un abanico amplio de posibilidades. De ahí la insignia de "posibilismo", nombre con el que quedó rotulada su obra.

Por su lado, Friedrich Ratzel llama la atención sobre las cuestiones económicas y políticas, buscando generalidades y regularidades en formulaciones teóricas más abstractas y especulativas que las de Vidal de la Blache. Ratzel procura investigar las influencias de la naturaleza sobre las personas, defiende que tales influjos son ejercidos de forma mediada a través de las condiciones económicas y sociales, siendo, para él, el medioambiente el soporte material de la vida; al mismo tiempo propone un análisis complejo y mediado por las interrelaciones entre sociedad, economía, territorio y Estado. Sin embargo, fue infelizmente rotulado como "determinista" ("environmentalist"). Es también uno de los principales autores que institucionalizaron la ciencia geográfica y es considerado fundador de la geopolítica, con sus discusiones sobre el territorio y el espacio vital.

Ambos investigadores contribuyeron así, con pionerismo y originalidad, en la búsqueda de un método de análisis adecuado a la complejidad de su objeto. Tanto La Blache como Ratzel son, además, proclives a los estudios empírico-inductivos, basados en la observación directa. Asimismo, buscaron la descripción y la catalogación, establecieron comparaciones y construyeron tipologías para sus investigaciones. Tuvieron también una mirada muy avanzada de los procesos sociales en torno a la producción y a la reproducción material de la vida, junto a una osada línea general de pensamiento que asume la idea de libertad de acción humana para moldar el espacio.

Por otra parte, sus propuestas también presentan muchas limitaciones, como, por ejemplo, cuando conciben el mundo de manera no interrelacionada o no interconectada; es decir, como un "paisaje" recipiente, perspectiva en la cual los objetos se vienen a localizar atomizados, en condiciones de existencia independientes o apartados entre sí. De forma semejante, la corriente teórica de la localización adoptará ese mismo enfoque, conforme veremos más adelante.

Otra línea de investigación obligatoria que debe ser recordada, aunque sea brevemente, es la denominada Escuela de Chicago. A partir del liderazgo intelectual de Robert Park, esta corriente elaboró una estructura teórica y empírica según una perspectiva que abordaba el fenómeno urbano conceptualizándolo como una comunidad ecológica. La ciudad era asumida como un laboratorio para la investigación de la complejidad de la vida social, a partir de la acción y del comportamiento individual. Se trató de una suerte de interpretación espacializada de la relación individuo-comunidad, generadora de un ordenamiento social según ciertos 
padrones locacionales, lectura que se daba a la par de un estudio de las relaciones centro-periferia específicas. Entre las concepciones de la ecología humana de esta escuela destacan los procesos de competición, integración, adaptación, cohesión y estabilidad social en el espacio urbano.

Es posible afirmar que las tres visiones que hasta aquí rescatamos logran compartir una visión de las relaciones interpersonales en cierto recorte espacial. Estas distintas corrientes poseen concepciones que pueden ser denominadas, en cierto modo, como materialistas, ya que buscan investigar las acciones humanas de forma personal o individualizada, en el contexto de la reproducción material. No obstante, su énfasis no fue puesto en la sociedad (ni en sus contradicciones), sino en el estudio de la comunidad.

Las tres corrientes pecarían también por su filiación al individualismo metodológico, adoptado en sus análisis. De forma general, los autores sintetizados anteriormente discutirán las tensiones contradictorias entre libertad individual y control social, o mejor aún, las condiciones de libertad para la acción individual que intenta cambiar su ambiente.

Ahora bien, procurando criticar la hegemonía teórica de esas tres visiones, algunos autores apuntarán importantes cuestiones sobre la reproducción social en el espacio urbano. Esta concepción crítica del ambiente construido, y disputado por las distintas fracciones de clases sociales, será central para los debates que abordaremos más adelante sobre la corriente crítica que se concentra en la producción social del espacio.

Conforme afirmamos más arriba, desde 1968, pero sobre todo a partir de las contribuciones de Lefebvre, Castells y Harvey, se busca entender la producción social del espacio y la dimensión urbano-regional de los procesos desiguales de desarrollo capitalista en contextos territoriales y escalares específicos como procesos dinámicos, históricos y contradictorios. Estos aportes encierran un largo y fructífero ciclo de elaboraciones teóricas e investigaciones empíricas entre 1968 y fines de los ochenta.

A partir de los ańos noventa, esta situación cambia, y más de una vez en la historia reciente experimentamos distintas ondas donde predominan modelos importados que "interpretan" nuestra realidad. Existe una clara colonización del pensamiento, junto a una pérdida de creatividad intelectual, para reflexionar de manera autónoma y desde una perspectiva propia y de conjunto sobre nuestro continente y nuestros respectivos países. Estamos, como nunca, sometidos a los centros de formulación y difusión de ideas dominantes (modelos acabados y estandarizados promovidos por los centros académicos hegemónicos o por organismos internacionales, consultorías, gurúes nacionales o internacionales, etcétera).

La fragmentación del campo de conocimiento territorial-espacial, la hegemonía de los paradigmas conservadores, los desvíos promovidos por el debate de la "posmodernidad" y su propuesta para "derrocar las grandes narrativas", el avance de la razón neoliberal, entre otros factores, han dificultado la construcción de trayectorias sustantivas críticas de entendimiento de la realidad concreta (histórica, tensionada y disputada). Y junto con todo ello, se ha interrumpido el análisis de los procesos materiales de reproducción social en el ámbito de la reflexión sobre el proceso de 
desarrollo capitalista, sobre todo de las características particulares en la periferia del sistema.

En estas concepciones, existe poco espacio para la oposición, para los conflictos o para la interdependencia de los espacios, con sus actores y sujetos sociales. Se elabora un discurso sofisticado de estabilización social e institucional, sin la discusión de las bases materiales y de los procesos sociopolíticos contradictorios, como si hubiera prácticas (sociales) sin estructuras (sociales). Así, desde la década de 1990, partiendo de un análisis que concibe a las estructuras sin sujetos, corremos el riesgo de haber caído en representaciones de actores sin estructuras.

No obstante, este artículo parte de la idea de que las posibilidades para realizar una reconstrucción crítica están dadas. Enseguida, defendemos que es importante situar y jerarquizar el lugar teórico de cada una de las principales determinaciones de la producción social del espacio. Con la intención de resaltar los más decisivos enlaces "faltantes", o "más frágiles", para así desarrollar colectivamente una propuesta de reconstrucción, apuntamos algunas de las mediaciones teóricas e históricas que requieren una mejor reelaboración o recalificación.

\section{Las cuatro corrientes teóricas del "pensamiento espacial"}

En este apartado, de manera didáctica y sucinta, planteamos que es posible detectar cuatro grandes corrientes del pensamiento social sobre el espacio: en el primer grupo encontramos las interpretaciones de la "localización"; en el segundo, la de los diversos "neoinstitucionalismos"; en el tercero, las de la "producción social del espacio"; y, por último, en el cuarto, las del "pensamiento crítico latinoamericano". Si bien presentamos las distintas corrientes por grupo, es importante considerar que esos cuatro troncos teóricos no son homogéneos, sino que, por el contrario, cada uno de ellos presenta variados matices y linajes.

\section{La interpretación de la localización: elecciones situacionales individualizadas en un espacio pasivo}

La primera línea teórica analizada en este apartado es también aquella que fue precursora del campo de los estudios espaciales y, por tanto, la más conocida. Si bien no cabe aquí recordar sus detalles, sí podemos resaltar sus ideas centrales.

En este grupo de formulaciones, el espacio se presenta como un mero receptáculo o recipiente pasivo. Desde esta visión analítica, el territorio es tan solo una "variable más", un "dato" de la realidad o un sitio fijo, inerte y neutro, sin contexto histórico ni sociopolítico. Un mero paisaje pasivo o una plataforma, donde vienen a inscribirse los objetos, las unidades situacionales que son tratadas como cosas (actividades económicas, individuos, etcétera) que, por algún motivo, se desplazaron; y esto lleva a plantear que tales movimientos, tales desplazamientos, deberían ser estudiados. Además, todo ello bajo el supuesto de que en todo el recorrido de análisis existe una armonía social subyacente, lo que resultará, al final del proceso de acción de ajuste y asignación de recursos por el mercado, en una disposición óptima racionalizada de los entes socioeconómicos en el espacio. 
Estas interpretaciones se sustentan en el individualismo metodológico y en elecciones racionales y maximizadoras de ventajas exclusivas y privadas. De esta manera, y teniendo en cuenta las restricciones iniciales y una dotación de recursos que se inscriben en una superficie homogénea, se realizan cálculos que regulan la eficiencia de los desplazamientos de los actores, principalmente por la vía de los costos del transporte.

\section{Las variadas corrientes de los nuevos institucionalismos: la negligencia en relación con los conflictos y las asimetrías de poder}

Existe una enorme pluralidad y eclecticismo en los enfoques institucionalistas, lo que hace difícil sintetizarlos en pocas palabras. Un punto en común en los diferentes abordajes es la búsqueda de discutir las normas y reglas que puedan encuadrar los comportamientos y las interacciones humanas. En este sentido, existe un interés en definir las instituciones como arreglos sociales y normalizaciones que poseen cierta estabilidad y duración.

Sin embargo, desafortunadamente, en las últimas décadas se puede constatar cierto abuso, inclusive cierta banalización del término "institución”. Esta palabra, que pasó a ser exageradamente extendida y utilizada de forma muy generalizada en casi todas las temáticas de las ciencias sociales, pierde así su sentido específico. También se puede afirmar que parte de la producción intelectual institucionalista exagera en su concepción de la capacidad endógena de los actores sociales, noción según la cual dichos actores, de manera autónoma, engendran procesos virtuosos y germinativos de densidad social; y, además, que esas autonomías estimularían procesos identitarios y cooperativismos específicos en cada comunidad, a través de procesos de mutualidades, aprendizajes y adquisiciones diferenciales ventajosas en el acúmulo colectivo.

En el centro de las interpretaciones de esta corriente de producción intelectual está la noción de que las intensas interacciones colectivas de los recursos humanos, culturales, sociopolíticos e institucionales propiciarían redes de compromisos, lealtades, sentimientos de pertenencia, capaces de engendrar coherencias y contratos sociales adecuadamente situados y contextualizados. Es decir, en esta corriente son exaltados los sistemas de colaboración y las redes de compromiso solidario localmente constituidos. Todo pasaría a depender de la adecuada estructuración de normas y del mejor comportamiento de los actores (individuos en asociación) involucrados en un sistema de valores culturales, saberes, etcétera. A la existencia de conflictos y disputas se le da escaso énfasis en esta corriente.

\section{La interpretación del pensamiento crítico del Norte: los conflictos de interés en relación con la producción social del espacio}

Sobre la discusión de las dimensiones espaciales de la crisis, las obras seminales de Henri Lefebvre, David Harvey, Manuel Castells, Alain Lipietz, Doreen Massey, Neil Smith, Edward Soja, entre muchos otros, surgieron también en el contexto pos 1973 y procuraron crear nuevas formulaciones, renovando el andamiaje teórico-metodológico del debate sobre la producción social del espacio al proponer nuevas perspectivas y categorías de análisis. Sus investigaciones sobre la producción 
y reproducción social del espacio y del ambiente construido pusieron énfasis en las relaciones sociales, en las luchas de clases y en las articulaciones entre Estado y las distintas fracciones de capital interactuando en el espacio, como también en los procesos a través de los cuales los sujetos sociales en pugna producen socialmente el espacio y el ambiente construido. Es decir, el espacio es interpretado como una construcción disputada y contradictoria, y se procura analizar y revelar los conflictos de intereses que lo estructuran.

En esta visión, ningún recorte espacial puede ser concebido como un ente pasivo, fijo o como mero receptáculo, sin un contexto institucional y sin una moldura histórica. Se trata, entonces, de reflexiones sobre los procesos de estructuración del espacio, que es disputado entre actores, agentes y sujetos que forjan y transforman estructuras, que crean estrategias en determinados campos espaciales y arenas de lucha y de conflictividad.

En los últimos años podemos contar, entre los aportes más significativos, los de Bob Jessop, Neil Brenner, Jamie Peck, Erik Swyngedouw, Georges Benko, Allen J. Scott, Ash Amin, Michael Storper, junto con los de muchos otros autores que elaboran y polemizan sobre el papel complejo de la dimensión espacial en el capitalismo contemporáneo.

Por último, mencionamos también que el pensamiento de la geografía crítica, por cierto muy dinámico y básicamente anglosajón, prestó poca atención a las formaciones subdesarrolladas y dependientes del sistema capitalista en su periferia. Por lo tanto, es preciso estimular un diálogo entre los abordajes de producción social del espacio y el pensamiento crítico histórico-estructural latinoamericano.

\section{El pensamiento crítico latinoamericano sobre el funcionamiento del capi- talismo periférico: pensando desde el Sur las especificidades del proceso de subdesarrollo}

En América Latina se concibió y se perfeccionó, desde finales de la década de 1940, un rico y creativo patrimonio científico-académico-político para pensar histórica y concretamente las especificidades del proceso de subdesarrollo en la condición periférica.

Con bastante originalidad, las reflexiones que dieron cuerpo a ese patrimonio procuraron plantear, a partir del Sur, una concepción de un cierto capitalismo suratlántico periférico-subdesarrollado, específicamente latinoamericano. Este pensamiento parte de una interpretación cíclico-dinámica de la reestructuración espacio-temporal de la contradicción histórico-sistémica de las relaciones centro-periferia en la escala mundial (Brandão \& Souza, 2019). Siendo bastante crítico y amplio, este marco contribuyó en la formulación de una concepción robusta sobre las especificidades de la experiencia de avances materiales, de diferenciación social e industrialización en el Sur Global.

Esta cuarta corriente ofrece aportes fundamentales para pensar la base material de nuestras sociedades latinoamericanas y concebir la importancia de estudiar, de forma bien regulada, la dinámica de las estructuras (productivas, comerciales, tecnológicas, de consumo y de la lógica de la acumulación de capital, etcétera), como también la dinámica de los sujetos concretos (intereses y conflictos de las facciones de clase) (Brandão, 2018). 
Vale recordar que, especialmente en las décadas de 1970 y 1980, tuvimos robustos aportes provenientes del estructuralismo histórico latinoamericano, que dialogaron más directamente con la dimensión espacial. Necesitamos retomarlos. Entre ellos podemos citar los magníficos aportes de Alejandro Rofman, Paul Singer, Wilson Cano, Silvia Gorenstein, Blanca Ramírez, José Luis Coraggio y Carlos de Mattos, junto a muchos otros.

\section{Apuntes sobre las posibilidades de una reconstrucción teórico-metodológica crítica}

Después de identificar las corrientes teóricas que procuraron interpretar las cuestiones referidas a la dimensión espacial de los procesos sociales, sería importante reflexionar, desde una perspectiva crítica, respecto de aquellas con las cuales se deberían establecer diálogos prometedores.

Este ensayo argumenta que la primera corriente (localización) y la segunda (neoinstitucionalismos) no presentan mayores contribuciones para los análisis de las cuestiones de la elaboración conflictiva y relacional del espacio construido en la sociedad capitalista. No posibilitan investigaciones sobre las relaciones sociales de clase, intereses, poder, acción estatal, disputas y contradicciones que se producen en el espacio, capaces de posicionar las contiendas interclases (fracciones sociales) en el centro de sus abordajes. En cambio, la reconstrucción de las mediaciones teóricas e históricas y la adecuada articulación entre las dos últimas interpretaciones -producción social del espacio y pensamiento crítico latinoamericano- pueden representar una muy fructífera posibilidad de reposicionamiento teórico crítico y espacializado desde el Sur.

Este recurso dialógico que proponemos también se justifica a partir de las dos últimas corrientes señaladas, porque son las que presentan las mayores carencias, conceptualizables como "enlaces ausentes" y "puentes virtuales". Estos vacíos pueden ser solucionados, sin embargo, en un mutuo refuerzo y articulación entre el funcionamiento específico del capitalismo y la "organización espacial"; es decir, construyendo o calibrando las viejas y eternas conexiones necesarias de nuestra área.

A continuación, presentamos algunos intentos que buscan identificar $y$ "completar" teóricamente algunos ejes de análisis, como también enlaces y puentes frágiles o ausentes de esta agenda crítica que se mueve entre la economía política y la geografía crítica elaboradas desde el Sur. De esta manera, volvemos a las problemáticas más generales, que se encuentran en un nivel de mayor abstracción, a fin de resaltar algunos puntos fundamentales para esta construcción.

Encontramos en el presente amplias posibilidades para realizar una reconstrucción teórico-metodológica crítica. Esta propuesta podría informar y estructurar las disputas políticas hacia las acciones sociales en el ambiente construido, fortaleciendo los movimientos contestatarios, además de colaborar en la orientación de políticas públicas democráticas.

Enseguida, como parte de la propuesta de este artículo, presentamos el esquema teórico contenido en la Tabla 1 , donde se despliegan tres categorías -división social 
del trabajo, poderes transformadores del espacio social y planos/niveles analíticos-, cada una de ellas con cuatro elementos constituyentes.

TABLA I Categorías de análisis y elementos constituyentes de una mirada alternativa

\begin{tabular}{|l|l|l|l|l|l|}
\hline \multicolumn{2}{|c|}{ CATEgORÍAs } & \multicolumn{2}{|c|}{ ELEMENTOs CONSTITUYENTES DE UNA MIRADA ALTERNATIVA } \\
\hline $1^{\text {a }}$ & $\begin{array}{l}\text { Dimensiones espa- } \\
\text { ciales de la división } \\
\text { social del trabajo }\end{array}$ & $\begin{array}{l}\text { Interramifica- } \\
\text { ciones }\end{array}$ & $\begin{array}{l}\text { Interregiona- } \\
\text { lidades }\end{array}$ & Interurbanidades & $\begin{array}{l}\text { Interacciones rivales entre } \\
\text { proyectos de actores y } \\
\text { sujetos concretos }\end{array}$ \\
\hline $2^{\text {a }}$ & $\begin{array}{l}\text { Poderes transfor- } \\
\text { madores del espacio } \\
\text { social }\end{array}$ & Privado & Público & $\begin{array}{l}\text { Poder de resisten- } \\
\text { cia democrática }\end{array}$ & $\begin{array}{l}\text { Poder de los micropo- } \\
\text { deres }\end{array}$ \\
\hline $3^{\text {a }}$ & $\begin{array}{l}\text { Planos o niveles de } \\
\text { análisis }\end{array}$ & $\begin{array}{l}\text { Escalas espa- } \\
\text { ciales }\end{array}$ & $\begin{array}{l}\text { Niveles de } \\
\text { gobierno }\end{array}$ & $\begin{array}{l}\text { Ámbitos y arenas } \\
\text { de poder }\end{array}$ & $\begin{array}{l}\text { Reproducción social de } \\
\text { las prácticas de la vida } \\
\text { cotidiana }\end{array}$ \\
\hline
\end{tabular}

FUENTE: ELABORACIÓN PROPIA

Las cuatro dimensiones espaciales de la división social del trabajo

La división social del trabajo es la categoría explicativa básica de la investigación que tiene por objeto la dimensión espacial en el desarrollo capitalista. Tal categoría atraviesa todos los procesos de dicho desarrollo y está presente en todas las escalas espaciales. Más específicamente, la división social del trabajo es una expresión de la etapa alcanzada por el desarrollo de las fuerzas productivas, como también de las relaciones sociales. Es una categoría mediadora adecuada para el estudio de las heterogeneidades, jerarquías y especializaciones intra e inter en cualquier escala, sean estas regionales, nacionales o internacionales. Su movimiento constante altera, impone lógicas externas, adapta, distingue y expone estructuras y dinámicas, promoviendo continuamente la redefinición y la redistribución de agentes, actividades, circuitos, funciones, etcétera.

Los espacios regionales y urbanos son el resultado de las dinámicas que operan y producen la diferenciación social, como también generan tipos de especialización y de diversificación material. Así, los estudios deben concentrarse en investigar la base operativa, es decir, el locus espacial en que se materializan dichos procesos, junto con examinar los centros de decisión y los sujetos históricos que los determinan.

A continuación proponemos describir detalladamente las cuatro más decisivas dimensiones espaciales del proceso dinámico de división social del trabajo: i) las interramificaciones; ii) las interregionalidades; iii) las interurbanidades; y iv) las interacciones entre individuos. ${ }^{2}$

\section{i. Interramificaciones o intersectorialidad económica en la dimensión espacial y social del trabajo}

Bajo el esquema que sugerimos, es necesario analizar las estructuras productivas situadas en un espacio urbano-regional específico, entendiéndolas como una densa

2 Con el fin de aunar criterios y resaltar la idea de lo "inter", nos remitimos a estos cuatro conceptos del siguiente modo: interramificaciones; interregionalidades; interurbanidades e interacciones. 
y compleja red de interramificaciones o intersectorialidades económicas inherentes a la producción capitalista. Ese sistema de interramificaciones posee notables relaciones entre sectores y ramas en permanente interacción dinámica en el espacio, como también una pluralidad de subdivisiones y subsecciones con claras especificidades productivas, técnicas y económicas. Al mismo tiempo, las distintas interramificaciones en el proceso socioeconómico de producción, circulación, distribución y consumo producen manifestaciones territoriales muy distintas.

Estudiando la dimensión de la intersectorialidad, es posible analizar la reproducción social, como también la naturaleza y el poder de las fuerzas productivas/ propulsivas y de los sistemas sociales de producción estructurados en un determinado tiempo-espacio. Además, se logra aprehender el conjunto de relaciones y efectos encadenados y los cortes productivos que se tejen en condiciones técnicas y de mercado completamente distintas.

Asimismo, los sectores pueden ser identificados y calificados por su inserción específica en la estructura productiva según sus categorías de uso (bienes de consumo duraderos y no duraderos, bienes intermedios y de capital).

\section{ii. Interregionalidades en la dimensión espacial y social del trabajo}

Para entender esta dimensión de la división social del trabajo a partir de las orientaciones metodológicas de Harvey (2004) y Braudel (1984), es importante señalar que el plan analítico interregional debe tratar de enfocar la articulación, la cohesión y la integridad de los procesos que tienen lugar en determinado espacio, explicitando sus mecanismos de coordinación y regulación: lo que los autores señalados denominaron, respectivamente, coherencia estructurada y coherencia impuesta.

El proceso de apertura, articulación e integración de los mercados funda una determinada interregionalidad coaccionada por el proceso de intensificación de la competencia inter e intraterritorial. Las interdependencias y complementariedades interregionales se multiplican, lo que puede conducir a un aumento tanto de las potencialidades como de sus vulnerabilidades. En este movimiento cambian los núcleos dinámicos de comando que ejercen distintos tipos de atracción y dominación, generando al mismo tiempo puntos estratégicos, ejes y nudos de mayor o menor potencia reproductiva y capacidad de apropiación.

\section{iii. Interurbanidades en la dimensión espacial y social del trabajo}

Es también necesario analizar las estructuras que componen la interurbanidad, las relaciones y las interacciones entre los espacios sociourbanos y su posicionalidad en una división interurbana e intraurbana del trabajo social.

La investigación crítica posee la responsabilidad inherente de examinar los determinantes del crecimiento urbano, la hegemonía de las coaliciones armadas en el espacio urbano y su proyección desde el interior de la red urbana y del sistema regional de ciudades, proyección que se realiza tanto desde el espacio interior de la ciudad como también desde su hinterland (Brenner, 2019).

Los procesos urbanos no pueden ser estudiados ni comprendidos dejando fuera la complejidad que posee la reproducción social. El proceso urbano es 
producto-producido por la constante pugna de facciones de clases sociales y la consolidación de hegemonías y luchas contrahegemónicas (Brandão, 2012).

iv. Interacciones interpersonales/intercorporalidades/intersubjetividades y la pluralidad de los proyectos de vida de los sujetos concretos en la dimensión espacial y social del trabajo

Las interacciones entre sujetos representan el campo de las prácticas espaciales y de las políticas de escala (Smith, 2002), pues, como afirma Massey (2008), "el espacio es la dimensión de lo social: de la coexistencia contemporánea con los otros" (p. 32). Constituye aquel campo de contraposición de disputas, fuerzas y narrativas en la escala de las experimentaciones de la vida cotidiana, a la vez que es el locus en el que se confrontan proyectos, poderes que se definen en la acción, en el plano de la vida individual y colectiva. Es en estas interacciones en el espacio donde también las lógicas exógenas son confrontadas o interiorizadas en cada sujeto. Se trata de lo que Milton Santos (1987) denomina "espacio del ciudadano".

Lo que estamos proponiendo aquí es el análisis de las decisiones u omisiones de accionar o de reaccionar de otro modo. Es decir, el estudio de las actuaciones, de las intencionalidades, las motivaciones y los comportamientos en el plano de la vida cotidiana de la casa, del hogar, del barrio, de la familia; el análisis de cada cuerpo específico en su peculiar tela de la vida en el mundo urbano y de las fisuras que son construidas en los espacios de la emancipación.

\section{Los cuatro poderes transformadores del espacio social}

Aquí planteamos investigaciones o estudios que, en aproximaciones sucesivas, aborden en las más variadas escalas los proyectos sociopolíticos y económicos en disputa, para lograr así dimensionar los poderes de sus actores, agentes y sujetos. En esta perspectiva, el espacio social es asumido como un campo de relaciones de fuerzas en confrontación. De esta manera, sería fundamental dimensionar los cuatro poderes que desarrollamos a continuación, investigando en cada uno de ellos su capacidad de comando, sus espacios de maniobra, sus instrumentos, etcétera.

\section{i. La capacidad del poder privado para transformar el espacio}

Es imprescindible volver a realizar investigaciones profundas orientadas a dimensionar el poder de decisión privado, sobre todo el de las grandes unidades conglomeradas oligopólicas que comandan ventajas cruciales y que poseen un poder cada vez más asimétrico de encadenamiento de decisiones transformadoras sobre el espacio social. El poder privado es un agente privilegiado que actúa en cadena, teniendo, además, una desigual capacidad de inducción sobre otras decisiones, incluso sobre las del Estado. Se encarna en conglomerados gerenciales y financieros en la forma de masas concentradas de capital-dinero. Dichos conglomerados están orientados por la exploración de oportunidades rentables y por la gestión de portfolios, invadidas por los procesos de financiarización y de acortamiento del horizonte de las decisiones empresariales. El curso intersectorial de las trayectorias de valoración se subordina a las orientaciones financieras (valor en bolsa), a las que someten todas las operaciones comerciales, terciarias, agrícolas e industriales, subordinándose además 
a sus designios y a su disciplina. Es crucial estudiar sus formas de control en los grandes circuitos de producción y distribución, al igual que sus tecnologías y sus fuentes de abastecimiento, entre otros aspectos.

\section{ii. El poder del poder público para transformar el espacio}

Existe una ineludible urgencia en retomar desde una perspectiva crítica -y especialmente en la coyuntura contemporánea- la discusión del papel del Estado en cuanto categoría fundante y constitutiva del capitalismo. Incluso más, esto debe realizarse al lado y al interior del aparato de Estado, reconociendo que junto a él existe un vasto y denso entorno. Es decir, un conjunto diversificado de entidades y arenas de articulación de intereses que podríamos denominar como "institucionalidades paraestatales" o estatalidad (statehood), que se reescalonan espacialmente por otros modos y vías paralelas y/o adyacentes al centro del aparato de decisión estatal (Brenner, 2004).

Este debate, que podría ser designado como reescalonamiento espacial-territorial del Estado (state space), debería avanzar en la investigación acerca de cómo el Estado y las estatalidades se especifican y particularizan en las diversas escalas espaciales, niveles y ámbitos gubernamentales y arenas de poder, como apuntaremos más adelante.

iii. El poder de las fuerzas de resistencia democráticas y emancipadoras para transformar el espacio

Aquí buscamos colocar en debate el dimensionamiento del poder de las facciones subalternas que emprenden luchas reivindicatorias, de movilización, protesta y resistencia. En este sentido, nos interesa evaluar la real capacidad de las fuerzas contestatarias e insurgentes para promover e impulsar una unificación de las distintas luchas democráticas y emancipadoras.

Esta propuesta procura estudiar las formas tácticas y las estrategias que se producen en las distintas formas de expoliaciones socioterritoriales. En este sentido, es preciso que analicemos las contrarreacciones de las fuerzas emancipadoras y reivindicativas, experimentadas a escala del lugar, en su búsqueda permanente de justicia socioespacial.

La canalización de las energías de insatisfacción y de inconformismo se expresa en formatos variados. Necesitamos, por tanto, investigar minuciosamente las contrarreacciones de los sin derecho y de los sin voz. Allí es donde surgen lógicas organizativas portadoras de acciones y reacciones de resistencia, que son susceptibles o no de constituirse en coaliciones insurgentes o alternativas. Pero, específicamente, estas formas diversas de resistencia social requieren estudios espacializados.

\section{iv. El poder de los micropoderes para transformar el espacio}

Además de los tres poderes anteriores, no debemos olvidar la producción social del espacio por parte de los micropoderes o contrapoderes de baja visibilidad. Son poderes que se organizan, potencializan y contraponen a las nuevas tecnologías del poder, que buscan normativizar y disciplinar comportamientos. También sería importante analizar la dimensión simbólica y las narrativas; en suma, la contraposición de representaciones, la diversidad de formas de acción y las luchas en el plano étnico-cultural de la reproducción del vivir. Se trata, además, de espacio-tiempos 
variados: el de lo doméstico, el de la comunidad, y tantos otros, que pueden sostener una cadena permanente de contrarreacciones. Estos micropoderes tienen el potencial para engendrar mecanismos más descentralizados y democráticos de decisión, potencializando o anteponiéndose a las cadenas de reacción y a las decisiones hegemónicas antipopulares y antidemocráticas.

\section{Los cuatro planos o niveles de análisis}

Defendemos en ese artículo que existen los siguientes planos o niveles de análisis: i) escalas espaciales; ii) niveles de gobierno; iii) instancias, ámbitos y arenas de poder; y iv) reproducción social de las prácticas espaciales de la vida cotidiana. Estos cuatro niveles requieren estudios precisos de sus dinámicas, naturalezas y especificidades, y exigen ser analizados y, sobre todo, articulados mediante un abordaje de conjunto.

\section{i. El plano de las escalas espaciales}

La discusión de las escalas espaciales de los procesos es, sin duda, una tarea obligada para la aprehensión de las determinaciones "territorializadas" de los fenómenos sociales, que en las últimas décadas se legitimó como una categoría central en los estudios de la dimensión espacial del desarrollo capitalista (Brandão, Fernández \& Ribeiro, 2019).

Las escalas espaciales deben ser tomadas como planos analíticos y niveles de abstracción que pueden dar un sentido a la organización de la reproducción social de la vida. Consideramos aquí la escala en cuanto categoría analítica, a la vez que como categoría de praxis política. Sin duda, las escalas son intrínsecamente inexactas y dinámicas. Son instancias y entidades en las que la vida social se organiza y se reproduce, trascendiendo la mera representación cartográfica. Pero la escala es también escenario político y locus del ejercicio de la hegemonía. Ella delimita, diseña y recorta, en constante proceso de confrontaciones y por interacción/oposición, compromisos sociopolíticos en movimientos conflictivos y contingentes.

Así, la investigación sobre la dimensión espacial y escalar del proceso de desarrollo no puede ignorar las cuestiones fundamentales del poder, de las jerarquías y hegemonías, cultivando una perspectiva de estas problemáticas en las distintas escalas espaciales.

\section{ii. El plano de los niveles de gobierno}

En los países federalizados, entender el pacto territorializado de poder es fundamental para dialogar con la cuestión de las escalas espaciales. La posibilidad de compartir procesos decisorios respecto de problemas comunes y la capacidad de estructuración y organización sociopolítica de las escalas nacional y subnacionales necesitan ganar creciente centralidad, principalmente en países con profundas y múltiples desigualdades sociales y regionales. Desde esta óptica, el federalismo es asumido como acuerdos, arreglos, normatividades, nuevos pactos, entendimientos, tensionados por contratos sociales conflictivos que deben ser afrontados en sus particularidades, en sus variedades históricas y espaciales; es decir, con las especificidades que poseen los países y las regiones. 
Las problemáticas del federalismo envuelven generalmente el campo de observación de la escala nacional, pero también deberían incluir el de sus escalas subnacionales. El pacto federativo se arma en el contexto y en el recorte escalar nacional. El nivel nacional es espacio de poder, arena de constitución, enfrentamiento y concertación de intereses. Pero, al mismo tiempo, es un constructo histórico, sociopolítico y económico de alineamiento político mínimo de los proyectos sociopolíticos en disputa.

En ese contexto, es necesario analizar el juego federativo y la espacialidad del Estado, a través de su capacidad de constituir planos escalares articuladores de la acción pública que tengan upscaling, hacia arriba, pero también capacidad para promover movimientos de downscaling del aparato estatal, hacia abajo.

\section{iii. El plano de los ámbitos y de las arenas de poder}

Las diversas instancias, ámbitos y arenas por donde se estructuran y fluyen los poderes requieren ser seguidos por los análisis críticos, advirtiendo que son compartimentos muy diversificados en sus naturalezas y lógicas internas. Como afirma Dunham (1976), "la organización espacial de cualquier actividad socioeconómica o administrativa entrańa una red de relaciones sociales y espaciales relativamente estable que se ha definido para servir determinados intereses y alcanzar ciertos objetivos concretos" (p. 66).

Es necesario, además, investigar los arreglos institucionales, perseguir los canales de influencia, las legislaciones, competencias, atribuciones, deliberaciones, instrumentos, acuerdos, junto con los procedimientos de una parafernalia compleja de aparatos estatales, paraestatales, órganos de fiscalización, reglamentación y control, o estatalidades que gravitan en el entorno del Estado, analizando también los procesos y rituales de poder que se producen en el espacio social a partir de los poderes de la República. Por ejemplo, analizar la discusión de aquella política urbana o regional que pasó por el Legislativo y después por el Ejecutivo y que, sin embargo, también debe ser comprendida en profundidad y detalle en el paso a paso de su recorrido, por las distintas dimensiones que conllevan los trámites en el ámbito del poder Judicial.

iv. El plano de la reproducción social de las prácticas espaciales de la vida cotidiana Este nivel o plano de análisis es esencial, pues es allí donde se desarrolla lo mundano de la tela fina del tejido social. En él es importante mirar las prácticas espaciales concretas en el contexto de esa esfera fundamental (inmediata, empírica y pragmática) de las rutinas de reproducción de la vida cotidiana. Es en la cotidianidad como locus del pragmatismo, de las carencias y de las preocupaciones, donde se formulan las respuestas inmediatas a situaciones singulares muy concretas, y lo que ahí se afirma es la "necesidad de buscar respuestas prácticas, condicionadas por las circunstancias sociales (...) en una superficialidad empírica” (Costa, 2001, p. 36).

Además, en el plano del "mundo de la vida" se tejen las intersubjetividades culturales y las expresiones de los derechos y de las libertades individuales, con sus demandas y sus lógicas propias. En este plano de análisis, las prácticas espaciales tienen el potencial para cambiar rutinas cotidianas de la vida social y construir otras formas de reciprocidad, distribución, que no necesariamente son las de mercado. 


\section{El desafío de hacer dialogar el esquema teórico propuesto con el pensamiento socioespacial crítico desde el Sur: algunos apuntes finales para una agenda de investigaciones}

El esquema teórico propuesto, sintetizado en la Tabla 1, buscó apuntar algunas dimensiones y elementos para, a partir de allí, montar una mirada alternativa a la dominante. En este sentido, si pretendemos contribuir en la construcción de un conocimiento contrahegemónico y desde el Sur -que se enfoque en la condición subordinada periférica en el contexto de las estructuras de poder globales-, se vuelve inevitable colocar en la propuesta algunos desdoblamientos y desafíos teóricos. El fin que aquí buscamos es claro: avanzar en un abordaje consistente y dinámico sobre el estudio de la producción social del espacio en América Latina.

En el Sur, el pensamiento crítico latinoamericano debe ser comprendido como un locus privilegiado de enunciación de la condición particular de la periferia capitalista en su posicionalidad específica; pero también debe ser aprovechado como una potencial fuente de inspiración, sobre todo a partir de su método de investigación original, que piensa estructural e históricamente los procesos sociales, políticos y económicos (Brandão \& Siqueira, 2020).

Ese legado científico precisa ser criticado y desafiado, para, después de ello, avanzar con su abordaje específico sobre las relaciones de clase y del Estado, como también sobre las relaciones de poder internacionales, y vencer cierto nacionalismo metodológico que el pensamiento histórico estructural aún posee. Requerimos desarrollar mejor los análisis comparativos y relacionales, además de ampliar su concepción escalar hacia una multiescalar. Igualmente, sería interesante analizar las interconexiones con otros espacios y cultivar una mirada más dinámica y en múltiples escalas espaciales. Sobre todo, es necesario perfeccionar sus planteamientos, "subiendo" escalarmente a las dinámicas geopolíticas, geoeconómicas y geoculturales, pero, al mismo tiempo, "bajando" a la escala de la vida cotidiana de las personas. Asimismo, este trabajo de recalibración debería tener mayor flexibilidad "lateral"/"horizontal", para estar así más abierto a pensar las coyunturas reales y concretas. Ello puesto que es en lo real y concreto donde se condensan las contradicciones en un periodo breve de tiempo, es decir, en escalas temporales o en temporalidades más "cortas".

Ese rico pensamiento permite tornar más maleable y flexible sus abordajes, actualizando tanto sus categorías de estilos de desarrollo como la de heterogeneidades estructurales, y avanzando así en una concepción más dinámica de las recombinaciones de los elementos de modernización y atraso. Esos elementos están siempre presentes, quedando yuxtapuestos y articulados a lo largo del tiempo en los espacios de la profunda heterogeneidad latinoamericana. De ahí la necesidad de buscar aprehender esas constelaciones de formaciones socioespaciales híbridas, que coexisten e interactúan en complejas articulaciones de lo arcaico y lo moderno.

El contexto periférico se compone, justamente, de esas estructuras malformadas o deformadas a lo largo del proceso histórico, configurando espacios vulnerables y sujetos a decisiones basadas en procesos de heteronomía, generalmente sometidos a las reglas impuestas por las relaciones imperiales que se expanden desde la escala mundial. 
En la Tabla 1 propusimos la evaluación de tres categorías centrales: las dimensiones de la división social del trabajo; de los poderes transformadores del espacio; y de los planos o niveles de análisis. De esta manera, propusimos estudiar en detalle, mediante investigaciones profundas, las divisiones sociales del trabajo y los poderes concretos y asimétricos. Esta propuesta coloca el foco en estas categorías porque es allí donde se engendran los "espacios periféricos" y donde, además, se producen las destituciones de renta, propiedad, derechos y poder de la mayoría (Brandão, 2016). Y es también el lugar donde se expresan las inmensas y profundas desigualdades regionales y las características del complejo proceso de urbanización, expansivo y precarizado.

Cada una de aquellas tres dimensiones y sus cuatro elementos constituyentes requieren ser examinados a partir del Sur. Persiguiendo ese fin, es pertinente preguntarse sobre cuáles son las características distintivas de la región latinoamericana, tomando como base, en primer lugar, las cuatro dimensiones espaciales de la división social del trabajo:

1. Interramificaciones de las estructuras productivas: en esta parte del mundo se presentan poco densas, interrumpidas y desconectadas en sus interrelaciones dentro de las redes, circuitos y cadenas extrarregionales de acumulación. Pero además, y sobre todo, estas estructuras están sometidas a decisiones de carácter exógeno, pues las subdivisiones de las ramas económicas con mayor valor agregado, las relaciones intersectoriales, el contenido tecnológico y los sectores líderes con mayor capacidad para arrastrar otros sectores, son de propiedad extranjera. Por estas razones, es importante ampliar la agenda de investigaciones de los grupos económicos y de sus conglomerados, junto con estudiar los circuitos regionales de acumulación en las diferentes regiones del continente.

2. Interregionalidades de las dependencias y complementariedades entre los espacios regionales: precisamos evaluar en el sur del mundo la articulación, la cohesión y la integridad de los procesos, explicitando sus mecanismos de coordinación y regulación. Ello permitiría un mapeo relacional de potencialidades, vulnerabilidades, jerarquías, puntos, ejes y nudos estratégicos de mayor o menor potencia reproductiva y capacidad de apropiación en el contexto de las relaciones interregionales.

3. Interurbanidades: necesitamos considerar de manera relacional las distintas "máquinas de crecimiento urbano" del subcontinente, pensando las coaliciones y los sujetos productores de espacios intra e interurbanos, a fin de realizar el análisis de los centros de decisión desde dentro de las redes urbanas, desde el espacio interior de la ciudad y su hinterland. Cabría destacar que las redes de ciudades latinoamericanas presentan muy específicas tasas de crecimiento, informalidad, junto con una combinación de formas modernas y atrasadas, en contextos de estructuras de urbanización y de ocupación muy precarizadas.

4. Interacciones rivales interpersonales: en estas dinámicas los sujetos confrontan sus proyectos en el plano de la vida tanto individual como colectiva, según distintas actuaciones, intencionalidades, motivaciones y comportamientos en 
el contexto de la vida cotidiana, ya sea de la casa, del hogar, del barrio, de la familia, etcétera, que en esta parte del mundo operan de modo bastante diferente de aquellas de los países desarrollados.

En cuanto a la segunda categoría central, poderes transformadores del espacio social, se podrían proponer las siguientes cuestiones para pensar América Latina. Analizamos cada uno de los cuatro poderes:

1. Poder privado: es preciso pensarlo en la forma de "unidades dominantes" que accionan cadenas de decisiones en el ambiente macroeconómico de vulnerabilidades tecnológicas y financieras con bajo poder de transformación estructural. Son conglomerados gerenciales mercantiles, que alojan su capital-dinero sobre todo en la órbita de la circulación y explotan oportunidades rentables en el capitalismo dependiente de forma subordinada en las cadenas globales de valor.

2. Poder público: es importante indagar en profundidad la naturaleza de la estatalidad periférica, junto con estudiar el reescalonamiento espacial-territorial del Estado, las particularidades de las relaciones Estado-Sociedad (statecraft), para así investigar las modalidades de organización institucional y espacial del poder público en contextos subdesarrollados.

3. Poder de las fuerzas contrahegemónicas: se requiere analizar las fuerzas de resistencia democrática y emancipadoras, realizando un balance y dimensionamiento de la fuerza organizativa de los movimientos sociales y el poder de las facciones subalternas, y avanzar sobre sus luchas específicas en los espacios heterogéneos rurales-urbanos-regionales de América Latina.

4. Micropoderes: necesitamos estudiar la especificidad de los espacios nacionales y regionales de los países periféricos. ${ }^{3}$ Es importante observar aquí las narrativas, contraponiendo sus representaciones, sus espacios de contestación y disputa, teniendo en cuenta la diversidad de formas de acción y lucha de los destituidos de derechos.

Finalmente, con respecto a la tercera categoría, planos o niveles de análisis, en diálogo con las especificidades del espacio latinoamericano, es crucial investigar:

1. El plano de las escalas espaciales: en cuanto producciones sociales en contextos nacionales atravesados por la heteronomía, con baja autonomía de decisiones.

2. El plano de los niveles de gobierno: para entender el pacto territorializado del poder y el enfrentamiento de problemas comunes, sobre todo en espacios subnacionales urbano-regionales precarizados, evaluando las capacidades financieras, burocráticas y técnicas del Estado para aplicar la gobernanza multinivel y los movimientos adecuados de upscaling y downscaling.

3 Hay una vasta literatura sobre interacciones rivales, prácticas espaciales de la vida cotidiana y micropoderes en América Latina, destacando la disponible en el sitio del Consejo Latinoamericano de Ciencias Sociales (clacso) (www.clacso.org). 
3. El plano de los ámbitos y arenas de poder: para concebir las diversas instancias, ámbitos y arenas por donde se estructuran y fluyen los poderes, comprendiendo sus agendas, sus arreglos institucionales, sus acuerdos, etcétera, ubicados según compartimentos muy diversificados en sus naturalezas propias y lógicas internas, examinando sus especificidades en las formaciones dependientes.

4. El plano de las prácticas espaciales de la vida cotidiana en la heterogeneidad latinoamericana: para comprender, en cada coyuntura particular, las rutinas de reproducción de la vida cotidiana. Estudiar las intersubjetividades, los proyectos individuales, desarrollados en ambientes de extremada desigualdad, junto con las disputas entre dominación y emancipación en los espacios del capitalismo periférico.

En suma, sería deseable aplicar una mirada holística, dialógica y equilibrada, que de forma estructural, histórica y dinámica, intente evaluar y medir las fuerzas para transformar la situación latinoamericana en cada una de las dimensiones y perspectivas aquí levantadas.

A lo largo de este trabajo buscamos sistematizar algunas tentativas de interpretación del papel del espacio, sea desde el gran enfoque estructural como también desde las dinámicas sociales. Además, hemos defendido que disponemos de un precioso patrimonio intelectual acumulado de reflexiones sobre los procesos dinámicos y conflictivos de producción del espacio entre agentes y sujetos que forjan y transforman estructuras, estrategias y arenas de lucha. Ese legado intelectual nos enseña cómo el rescate de la problemática de la reproducción de las clases sociales es crucial, sobre todo al momento de intentar entender la dimensión urbano-regional de los procesos desiguales de desarrollo capitalista en contextos territoriales y escalares específicos.

Intentamos también, desde una perspectiva crítica, situar y jerarquizar, según el lugar teórico de cada una de las principales determinaciones de la producción social del espacio, los más decisivos enlaces "faltantes" o "más frágiles".

Por último, junto con todo lo anterior, reclamamos la necesidad de reconocer la rica y poco explotada herencia del Sur, y al mismo tiempo, la urgencia de acompañar el avance de la frontera del conocimiento crítico del Norte. Cabría construir robustas y más definidas mediaciones teóricas e históricas que interpelen las transformaciones geoeconómicas, geopolíticas y geoculturales del capitalismo del siglo xxi, sobre todo en el actual momento de profunda y multidimensional crisis, que avanza junto con un marcado proceso de neoliberalización radical posdemocrática. Simultáneamente, apremia buscar y sostener la construcción de mediaciones a partir de las especificidades de los espacios periféricos, subdesarrollados, dependientes e imperializados del Sur, con el objetivo de pensar la producción social del espacio en todas sus dimensiones. Por último, sería bueno dejar claro que se tiene plena consciencia de que un conjunto de tan complejas problemáticas requiere investigaciones colectivas, reclamando además un posicionamiento crítico y situado en el Sur del mundo. 


\section{Agradecimientos}

El autor agradece las lecturas atentas, provocativas y las precisas sugerencias de los evaluadores, que aportaron mejoras substantivas a la versión previa de este artículo. Estoy en deuda con Martin Scarpacci por las interlocuciones, traducción y revisión de las distintas versiones y las discusiones de varias ideas y mejor forma de exponerlas. El presente trabajo recibe beca de Productividad en Pesquisa (PQ) del Consejo Nacional de Desarrollo Científico y Tecnológico de Brasil (cnpq).

\section{Referencias bibliográficas}

Brandão, C. (2012). Território e desenvolvimento: as múltiplas escalas entre o local e o global. Editora da Unicamp.

Brandão, C. (2016). Espaços da destituição e as políticas urbanas e regionais no Brasil: uma visão panorâmica. Nova Economia, 26(núm. especial), 1097-1132. http://dx.doi. org/10.1590/0103-6351/3961

Brandão, C. (Org.). (2018). Teorias epoliticas do desenvolvimento latino-americano. Contraponto/ Centro Celso Furtado.

Brandão, C., Fernández, V. R. \& Ribeiro, L. C. Q. (Orgs.). (2019). Escalas espaciais, reescalonamentos e estatalidades: liçōes e desafios para América Latina. LetraCapital.

Brandão, C. \& Siqueira, H. (2020). Interpretations of underdevelopment in Brazil. En Oxford Research Encyclopedia of Latin American History. Oxford University Press. https://doi. org/10.1093/acrefore/9780199366439.013.866

Brandão, C. \& Souza, M. B. (2019). Particularidades del capitalismo periférico brasileño y sus recientes oleadas de neoliberalización (1995-2018). Semestre Económico, 22(50), 2345. https://doi.org/10.22395/seec.v22n50a2

Braudel, F. (1984). Civilización material, economía y capitalismo. Alianza.

Brenner, N. (2004). New state spaces: urban governance and the rescaling of statehood. Oxford University Press.

Brenner, N. (2019). New urban spaces: urban theory and the scale question. Oxford University Press.

Capel, H. (1981). Filosofía y ciencia en la geografía contemporánea. Una introducción a la geografía. Barcanova.

Castells, M. (1972). La question urbaine. François Maspero.

Castro, I. E., Costa Gomes, P. C. \& Lobato Corrêa, R. (Orgs.). (1995). Geografia: conceitos e temas. Bertrand Brasil.

Coraggio, J. L. (1987). Territorios en transición. Editorial Ciudad.

Costa, L. C. (2001). A estrutura da vida cotidiana: uma abordagem através do pensamento lukacsiano. Emancipação, 1(1), 33-57.

De Mattos, C. A. (1989). Reestructuración social, grupos económicos y desterritorialización del capital. El caso de los países del Cono Sur. Revista EURE - Revista de Estudios Urbano Regionales, 16(47), 61-90. http://www.eure.cl/index.php/eure/article/view/1050 
Dunham, D. M. (1976). Intereses de grupos y estructuras espaciales: algunas proposiciones teóricas. Revista EURE - Revista de Estudios Urbano Regionales, 5(13), 61-78. http:// eure.cl/index.php/eure/article/view/875/729

Fernandes, F. (1972). Capitalismo dependente e classes sociais na América Latina. Zahar.

Furió, E. (1996). Evolución y cambio en la economía regional. Ariel.

Gottdiener, M. (1997). A produção social do espaço urbano. Edusp - Editora da Universidade de São Paulo.

Haesbaert, R. (2019). Regional-Global: dilemas de la región y de la regionalización en la geografía contemporánea. Consejo Latinoamericano de Ciencias Sociales (CLACSO).

Harvey, D. (1973). Social Justice and the City. Edward Arnold.

Harvey, D. (2004). El nuevo imperialismo. Akal.

Lefebvre, H. (1968). Le Droit à la ville. Anthropos.

Lencioni, S. (1999). Região e geografia. Edusp - Editora da Universidade de São Paulo.

Massey, D. (2008). Pelo espaço. Bertrand Brasil.

Méndez, R. (1997). Geografía Económica. La lógica espacial del capitalismo global. Ariel.

Moraes, A. C. R. (2000). Geografia: pequena história crítica (20a ed.). AnnaBlume.

Paasi, A., Harrison, J. \& Jones, M. (Eds.). (2018). Handbook on the geographies of regions and territories. Edward Elgar Publishing.

Riffo, L. (2013). 50 años del ILPES: evolución de los marcos conceptuales sobre desarrollo territorial. Serie Desarrollo Territorial, (15). [Lc/L.3593]. Naciones Unidas - Comisión Económica para América Latina y el Caribe (CEPAL). https://www.cepal.org/sites/default/files/ publication/files/7248/S1303593_es.pdf

Rofman, A. B. (1974). Dependencia, estructura de poder y formación regional en América Latina. Siglo Veintiuno.

Santos, M. (1987). Espaço do cidadão. Nobel.

Smith, N. (2002). Geografía, diferencia y las políticas de escala. Terra Livre, 18(19), 127-146. 